\title{
Light, Shadows and Surface Characteristics. The Multispectral Portable Light Dome
}

\author{
Lieve Watteeuw ${ }^{1 *}$, Hendrik Hameeuw ${ }^{1,3}$, Bruno Vandermeulen ${ }^{1,}$ Athena Van \\ der Perre ${ }^{1,3}$, Vanessa Boschloos ${ }^{3}$, Luc Delvaux ${ }^{3}$, Marina Van Bos ${ }^{4}$, Marc \\ Proesmans ${ }^{2}$, Luc Van Gool ${ }^{2}$
}

1 KU Leuven, Faculty of Arts, Blijde Inkomststraat 21, 3000 Leuven (Belgium),

2 KU Leuven, VISICS, ESAT/PSI, Arenberg 10, 3001 Leuven (Belgium).

3 Royal Museums of Art and History (RMAH), Antiquity department, Jubelpark 10, 1000 Brussels (Belgium).

$4 \quad$ Royal Institute for Cultural Heritage, Jubelpark 1, Brussels ( Belgium)

* Lieve Watteeuw, tel.: 0032 486834285, lieve.watteeuw@arts.kuleuven.be

Keywords: multispectral imaging; photometric stereo; conservation; (mobile) documentation; non-invasive techniques; art-technical studies

The interplay of changing light directions and shades play a central role for an examiner to explore and understand the material characteristics of a surface. A researcher or restorer/conservator is in the first place trained to apply this with the naked eye using white (VIS) light. Since several decades multispectral imaging (UV, IR and near-IR) has proven its value for the field of cultural heritage studies, especially as one of its main advantages lies in the non-invasive / non-destructive character of this approach. In art technical research, conservation science and archaeology, applying these different spectra enables a precise examination and identification of colours and pigments, the visualisation of underlying features, and the identification of varying materials or surface conditions. A shortcoming of these approaches, however, is the absence of geometrical information on the surface in these datasets and the userfriendliness of their acquisition and viewer systems.

Within the framework of the research projects RICH (University of Leuven) and EES (Royal Museums of Art and History, Brussels), a multispectral, multi-directional, portable and dome-shaped acquisition system has been developed in collaboration with the ESAT-VISICS research group of the University of Leuven. This Multispectral Portable Light Dome (PLD) consists of a hemispherical structure, with an overhead camera and LEDs emitting in five different parts of the electromagnetic spectrum, ranging from ultraviolet over visible to infrared light, regularly covering the dome's inside surface. With the associated software solution, virtual relighting and enhancements can be applied in a real-time, interactive manner. The dome extracts genuine $3 \mathrm{D}$ and shading information based on the principles of photometric stereo. This innovative approach allows for instantaneous alternations between the computations in the IR, R, G, B and UV light spectra. All visualization

modes are based on a single recording procedure, taking only a few minutes. They enable the detection of object surfaces even when difficult to access, the generation of

$2^{\text {nd }}$ International Conference on Innovation in Art Research and Technology, 21-25 March 2016, Ghent, Belgium 
2D+ models, and the life-like or visually enhanced interaction with the recorded artefact. This leads to a user-friendly and cost-effective methodology, applicable to a wide variety of heritage objects.

The MS system has been used by the RICH and EES projects for the research on medieval manuscript illuminations and fragile Egyptian objects. Imaging the characteristics of the undulating parchment, the pictorial layers, the laid gold, the faded inscriptions and the tactile characteristics of the art and physical objects reveals to scholars and conservators the 'hand', the techniques and the materials of the original artists and artefacts. Preliminary results have shown this system can 1) document and measure the 3D surface structure of the objects, 2) the sequence of the writing, 3) the underdrawing, 4) the preparation of gilded layers, 5) the brushstrokes applying the different mineral and organic materials, 6) the finishing of the pictorial touch with glazes and pen work, 7) re-visualize faded pigments and inscriptions, 8) examine the MS results in combination with the actual relief characteristics and properties of the physical object. Besides supporting questions of attribution, changes and decay of the material characteristics can be monitored, supporting decision-making in the conservation protocol.

Reflectance Imaging for Cultural Heritage (RICH) is a project from Illuminare (Centre for the Study of Medieval Art, KU Leuven), financed by the Hercules Foundation 2012-2015 (Flemish government) Project AKUL/11/03, site: http://www.illuminare.be/rich_project.

The Egyptian Execration Statuettes project (EES) is financed by the Brain-be Pioneer programme of the Belgian Science Policy Office-BELSPO (project BR/121/PI/EES): http://www.kmkgmrah.be/conservation-ir-uv-and-3d-imaging-egyptian-execration-statuettes, and supported by the BELSPO Interuniversitary Attraction Poles Programme Greater Mesopotamia - Reconstruction of its Environment and History (GMREH) (Project IAP 7/14): http://www.greatermesopotamia.be.

Based on the Portable Light Dome, see H. Hameeuw, G. Willems, Akkadica. 2011; 132, pp.163-178.

L. Watteeuw, B. Vandermeulen, J. Van der Stock, P. Delsaerdt, S. Gradmann, F. Truyen, M. Proesmans, W. Moreau, L. Van Gool, in: Paper Conservation. Decisions \& Compromise. ICOM-CC graphic documents working group interim meeting, (Eds: L. Watteeuw, C. Hofmann). ICOM- CC \& Austrian National Library, Vienna, 2013, pp.140-141. For a particular resultRoman mummy portraits, see A. Van der Perre, H. Hameeuw, in: Sarcophages. Sous les étoiles de Nout. (Eds : L. Delvaux, I. Therasse ) Racine, Brussels, 2015, pp.164-165.

$2^{\text {nd }}$ International Conference on Innovation in Art Research and Technology, 21-25 March 2016, Ghent, Belgium 